\title{
SUMS OF CUBES IN POLYNOMIAL RINGS
}

\author{
L. N. VASERSTEIN
}

ABSTRACT. For any associative ring $A$ with 1 of prime characteristic $\neq 0,2$, 3 , every element of $A$ is the sum of three cubes in $A$.

For any ring $A$, let $w_{3}(A)$ denote the least integer $s \geq 0$ such that every sum of cubes in $A$ is a sum of $s$ cubes in $A$. If no such $s$ exists, $w_{3}(A)=\infty$ by definition.

For example, when $A=\mathbb{Z}$, the integers, it is known [2] that $4 \leq w_{3}(\mathbb{Z}) \leq 5$.

In this paper, we study $w_{3}(A)$ for $A=F[x]$, the polynomial ring in one variable $x$ with coefficients in a field $F$. It is easy to see [4] that every polynomial in $F[x]$ is a sum of cubes, if and only if $\operatorname{char}(F) \neq 3$ and $\operatorname{card}(F) \neq 2,4$. If this is the case, $w_{3}(F[x])$ coincides with the least $s$ such that $x$ is the sum of $s$ cubes in $F[x]$. Moreover, in this case, every element of any associative $F$-algebra $A$ with 1 is the sum of $w_{3}(F[x])$ cubes in $A$; in particular, $w_{3}(A) \leq w_{3}(F[x])$.

The formula

$$
(x+1)^{3}-2 x^{3}+(x-1)^{3}=6 x
$$

shows that $w_{3}(A) \leq 4$ for any associative ring $A=6 A$ with 1 . In particular, $w_{3}(F[x]) \leq 4$ for any field $F$ with $\operatorname{char}(F) \neq 2,3$. By [4], $w_{3}(F[x]) \leq 4$ also in the case when $\operatorname{char}(F)=2$ and $\operatorname{card}(F) \neq 2,4$. When $\operatorname{card}(F)=2,4$, formulas on p. 63 of [3] show that $w_{3}(F[x]) \leq 5$. These formulas together with the formulas

$$
\begin{aligned}
(x y+s)^{3}+ & (x y+s+1)^{3}+\left(x+s y^{2}\right)^{3}+\left(x+(1+s) y^{2}\right)^{3}=x\left(y+y^{4}\right) \\
\left(x^{2} y^{2}\right. & \left.+x\left(y+y^{4}\right)+1+s+y+y^{8}+y^{10}\right)^{3} \\
& +\left(x^{2}\left(1+y^{2}\right)+x\left(y+y^{4}\right)+s+y+y^{2}+y^{10}\right)^{3} \\
& +\left(x^{2} y^{2}+x\left(y+y^{4}\right)+s+y+y^{4}+y^{8}+y^{10}\right)^{3} \\
& +\left(x^{2}\left(1+y^{2}\right)+x\left(y+y^{4}\right)+s+y+y^{2}+y^{4}+y^{10}\right)^{3} \\
= & x\left(y+y^{4}\right)+y+y^{2}
\end{aligned}
$$

where $2=0$ and $s^{2}+s+1=0$, show that $w_{3}(F[x]) \leq 4$ in the case when $\operatorname{card}(F)=4$.

Received March 31, 1989; revised February 15, 1990.

1980 Mathematics Subject Classification (1985 Revision). Primary 11 P05, 11 C08.

Research supported in part by NSF grant DMS 86-20428. 
On the other hand, it is clear that $w_{3}(F[x])=1$ when $\operatorname{char}(F)=3$, and that $w_{3}(F[x]) \geq 3$ when $\operatorname{char}(F) \neq 3$.

Our first easy result (which was obtained by the author in July of 1987 and was known to J.-P. Serre since April of 1982) is the following

Lemma 1. If $\operatorname{char}(F) \neq 3$ and there are nonzero $\alpha, \beta, \gamma \in F$ such that $\alpha^{3}+$ $\beta^{3}+\gamma^{3}=0$, then $w_{3}(F[x])=3$.

Proof. The formula

$$
\left(\alpha x+\beta^{2}\right)^{3}+\left(\beta x-\alpha^{2}\right)^{3}+(\gamma x)^{3}=\beta^{6}-\alpha^{6}-3 \alpha \beta \gamma^{3} x,
$$

where $x$ can be replaced by an arbitrary polynomial in $F[x]$, shows that $w_{3}(F[x]) \leq 3$.

Corollary 2. If $\operatorname{char}(F) \neq 0,3$ and $\operatorname{card}(F) \neq 2,4,7,13,16$, then $w_{3}(F[x])$ $=3$.

By more complicated computations, we will prove the following

Proposition 3. If $\operatorname{char}(F) \neq 3$, then the condition of Lemma 1 is equivalent to the following: $x$ is the sum of cubes of three polynomials in $F[x]$ of degree $\leq 4$.

J.-P. Serre knew this for polynomials of degree $\leq 2$ since April of 1982 . The case of degree $\leq 3$ was done by August 1987 independently by the author and David Hayes. In his letter of August 11, 1987, to Serre, Hayes wrote that the case of degree $\leq 4$ defeated him. The author obtained Proposition 3 with $2 F \neq F$ in September of 1987 . He thanks Serre for providing copies of relevant letters and useful suggestions.

This result leads one to wonder whether the converse of Lemma 1 is true. However J.-P. Serre wrote to the author on September 9, 1987, that he did not dare to conjecture anything himself even for $F=\mathbb{Q}$, the rational numbers, or $F=\mathbb{Z} / 7 \mathbb{Z}, \mathbb{Z} / 13 \mathbb{Z}$.

Computations with polynomials of degree $\geq 5$ are very complicated, and it is only after many hours of computations with computers, that the author obtained the following result.

Theorem 4. If $\operatorname{char}(F) \neq 0,3$ and $\operatorname{card}(F) \neq 2,4,16$, then $w_{3}(F[x])=3$. So every element of every associative $F$-algebra $A$ is the sum of three cubes in $A$.

Proof. By Corollary 2, we have only the cases $\operatorname{char}(F)=7,13$ to deal with. Modulo 13, we have

$$
\begin{gathered}
\left(-4 x+5 x^{2}+6 x^{3}+x^{4}\right)^{3}+\left(1+4 x+x^{2}-5 x^{3}-3 x^{4}+5 x^{5}\right)^{3} \\
+\left(-1+5 x-5 x^{2}+5 x^{3}+3 x^{4}-5 x^{5}\right)^{3}=x .
\end{gathered}
$$

Modulo 7, we have

$$
\left(x-3 x^{3}-x^{5}\right)^{3}+\left(-1-x-x^{3}+3 x^{4}-x^{6}\right)^{3}+\left(1-x-x^{3}-3 x^{4}+x^{6}\right)^{3}=x \text {. }
$$


Remarks. The cases $F=\mathbb{Q}$ and $\operatorname{card}(F)=16$ remain unresolved. The above equalities were found using Mathematica on Macintosh IIx. We work in one of the following cases: $F=\mathbb{Q}, \mathbb{Z} / 7 \mathbb{Z}$, or $\mathbb{Z} / 13 \mathbb{Z}$. In degree 5 , we proceed as follows. First, we set $d=a^{3}+b^{3}+c^{3}$ with

$$
\begin{aligned}
& a=a_{0}+a_{1} x+a_{2} x^{2}+a_{3} x^{3}+a_{4} x^{4}+a_{5} x^{5} \\
& b=b_{0}+b_{1} x+b_{2} x^{2}+b_{3} x^{3}+b_{4} x^{4}+b_{5} x^{5} \\
& c=c_{0}+c_{1} x+c_{2} x^{2}+c_{3} x^{3}+c_{4} x^{4}+c_{5} x^{5}
\end{aligned}
$$

Note that $d=d_{0}+d_{1} x+\cdots+d_{15} x^{15}$, where $d_{j}$ are polynomials in $a_{i}, b_{i}, c_{i}$. We want $d_{j}=0$ for $i \geq 2$ and $d \neq 0$. (For proving Theorem 4 , we want $d_{1} \neq 0$.) We assume that $\operatorname{char}(F) \neq 2,3$. By Proposition 3 , we can assume that one of the leading coefficients $a_{5}, b_{5}, c_{5}$ is not 0 . Since the condition of Lemma 1 does not hold, one of these coefficients must be 0 . Say, $a_{5} \neq 0$ and $c_{5}=0$. Then $b_{5}=-a_{5}$, because $d_{15}=0$. The condition $d \neq 0$ forces $c_{4} \neq 0$. By a linear invertible change of the variable $x$ and multiplying both sides of $d=a^{3}+b^{3}+c^{3}$ by a nonzero constant, we are reduced to the case when $a_{5}=1=-b_{5}, a_{4}=0, c_{4}=1$. Now $d_{15}=0 \neq d$.

The polynomial equations $d_{i}=0 \quad(7 \leq i \leq 15)$ allow us to exclude all unknown coefficients but $a_{3}, c_{0}, c_{1}, c_{2}, c_{3}$. The conditions $d_{6}=0, d_{5}=0, d_{4}=$ $0, d_{3}=0, d_{2}=0$ give a system of five polynomial equations for five unknowns $a_{3}, c_{0}, c_{1}, c_{2}, c_{3}$. Namely,

$$
\begin{aligned}
d_{6}= & -1 / 108+5 a_{3}^{3}+3 a_{3} c_{0}+3 c_{1}^{2} / 4-18 a_{3}^{2} c_{2}-3 c_{0} c_{2}+21 a_{3} c_{2}^{2} \\
& -8 c_{2}^{3}-12 a_{3} c_{1} c_{3}+15 c_{1} c_{2} c_{3}+63 a_{3}^{2} c_{3}^{2}+3 c_{0} c_{3}^{2}-159 a_{3} c_{2} c_{3}^{2} \\
& +99 c_{2}^{2} c_{3}^{2}-3 c_{1} c_{3}^{3} / 2+12 a_{3} c_{3}^{4}-21 c_{2} c_{3}^{4}-37 c_{3}^{6} / 4=0 \\
d_{5}= & 9 a_{3}^{2} c_{1} / 2+3 c_{0} c_{1} / 2-12 a_{3} c_{1} c_{2}+15 c_{1} c_{2}^{2} / 2-c_{3} / 12 \\
& -30 a_{3}^{3} c_{3}-3 a_{3} c_{0} c_{3}+3 c_{1}^{2} c_{3} / 4+117 a_{3}^{2} c_{2} c_{3}+6 c_{0} c_{2} c_{3} \\
& -150 a_{3} c_{2}^{2} c_{3}+63 c_{2}^{3} c_{3}-6 a_{3} c_{1} c_{3}^{2}+9 c_{1} c_{2} c_{3}^{2} / 2 \\
& +57 a_{3}^{2} c_{3}^{3} / 2+15 c_{0} c_{3}^{3} / 2-51 a_{3} c_{2} c_{3}^{3}+33 c_{2}^{2} c_{3}^{3} / 2 \\
& -15 c_{1} c_{3}^{4} / 2+57 a_{3} c_{3}^{5}-165 c_{2} c_{3}^{5} / 2-69 c_{3}^{7} / 4=0 \\
d_{4}= & a_{3} / 18+15 a_{3}^{4} / 4+3 a_{3}^{2} c_{0} / 2+3 c_{0}^{2} / 4-c_{2} / 12 \\
& -12 a_{3}^{3} c_{2}+3 c_{1}^{2} c_{2} / 4+21 a_{3}^{2} c_{2}^{2} / 2-3 c_{0} c_{2}^{2} / 2 \\
& -9 c_{2}^{4} / 4-6 a_{3}^{2} c_{1} c_{3}+3 c_{0} c_{1} c_{3} / 2+9 c_{1} c_{2}^{2} c_{3} \\
& -c_{3}^{2} / 3+42 a_{3}^{3} c_{3}^{2}-159 a_{3}^{2} c_{2} c_{3}^{2} / 2 \\
& +9 c_{0} c_{2} c_{3}^{2} / 2+81 c_{2}^{3} c_{3}^{2} / 2-3 c_{1} c_{2} c_{3}^{3} \\
& +6 a_{3}^{2} c_{3}^{4}+3 c_{0} c_{3}^{4} / 2-69 c_{2}^{2} c_{3}^{4} / 4-3 c_{1} c_{3}^{5} / 2-51 c_{2} c_{3}^{6} / 4-3 c_{3}^{8} / 2=0 ;
\end{aligned}
$$




$$
\begin{aligned}
& d_{3}=21 a_{3}^{3} c_{1} / 2+9 a_{3} c_{0} c_{1} / 2+c_{1}^{3}-33 a_{3}^{2} c_{1} c_{2}-3 c_{0} c_{1} c_{2} \\
& +63 a_{3} c_{1} c_{2}^{2} / 2-9 c_{1} c_{2}^{3}+a_{3} c_{3} / 3-135 a_{3}^{4} c_{3} / 4 \\
& -6 a_{3}^{2} c_{0} c_{3}+3 c_{0}^{2} c_{3} / 4-18 a_{3} c_{1}^{2} c_{3}-c_{2} c_{3} / 2 \\
& +129 a_{3}^{3} c_{2} c_{3}+9 a_{3} c_{0} c_{2} c_{3}+45 c_{1}^{2} c_{2} c_{3} / 2 \\
& -321 a_{3}^{2} c_{2}^{2} c_{3} / 2+63 a_{3} c_{2}^{3} c_{3}+9 c_{2}^{4} c_{3} / 4 \\
& +219 a_{3}^{2} c_{1} c_{3}^{2} / 2+9 c_{0} c_{1} c_{3}^{2} / 2-549 a_{3} c_{1} c_{2} c_{3}^{2} / 2 \\
& +333 c_{1} c_{2}^{2} c_{3}^{2} / 2-3 c_{3}^{3} / 4-209 a_{3}^{3} c_{3}^{3} / 2 \\
& +3 a_{3} c_{0} c_{3}^{3} / 2-9 c_{1}^{2} c_{3}^{3} / 4+393 a_{3}^{2} c_{2} c_{3}^{3} \\
& +9 c_{0} c_{2} c_{3}^{3} / 2-933 a_{3} c_{2}^{2} c_{3}^{3} / 2+171 c_{2}^{3} c_{3}^{3} \\
& +12 a_{3} c_{1} c_{3}^{4}-63 c_{1} c_{2} c_{3}^{4} / 2+42 a_{3}^{2} c_{3}^{5}-87 a_{3} c_{2} c_{3}^{5} / 2 \\
& -81 c_{2}^{2} c_{3}^{5} / 4-27 c_{1} c_{3}^{6} / 2+6 a_{3} c_{3}^{7}-45 c_{2} c_{3}^{7} / 2-9 c_{3}^{9} / 4=0 \text {; } \\
& d_{2}=-a_{3}^{2} / 9+9 a_{3}^{5} / 2+9 a_{3}^{3} c_{0}+9 a_{3} c_{0}^{2} / 2+27 a_{3}^{2} c_{1}^{2} / 4+3 c_{0} c_{1}^{2} \\
& +a_{3} c_{2} / 3-99 a_{3}^{4} c_{2} / 4-63 a_{3}^{2} c_{0} c_{2} / 2-15 c_{0}^{2} c_{2} / 4-18 a_{3} c_{1}^{2} c_{2} \\
& -c_{2}^{2} / 4+54 a_{3}^{3} c_{2}^{2}+36 a_{3} c_{0} c_{2}^{2}+45 c_{1}^{2} c_{2}^{2} / 4-117 a_{3}^{2} c_{2}^{3} / 2 \\
& -27 c_{0} c_{2}^{3} / 2+63 a_{3} c_{2}^{4} / 2-27 c_{2}^{5} / 4-135 a_{3}^{3} c_{1} c_{3} / 2-27 a_{3} c_{0} c_{1} c_{3} \\
& +252 a_{3}^{2} c_{1} c_{2} c_{3}+36 c_{0} c_{1} c_{2} c_{3}-621 a_{3} c_{1} c_{2}^{2} c_{3} / 2+126 c_{1} c_{2}^{3} c_{3} \\
& +5 a_{3} c_{3}^{2} / 6+549 a_{3}^{4} c_{3}^{2} / 4+108 a_{3}^{2} c_{0} c_{3}^{2}+9 c_{0}^{2} c_{3}^{2} / 2+9 a_{3} c_{1}^{2} c_{3}^{2} \\
& -5 c_{2} c_{3}^{2} / 4-639 a_{3}^{3} c_{2} c_{3}^{2}-270 a_{3} c_{0} c_{2} c_{3}^{2}-63 c_{1}^{2} c_{2} c_{3}^{2} / 4 \\
& +1107 a_{3}^{2} c_{2}^{2} c_{3}^{2}+333 c_{0} c_{2}^{2} c_{3}^{2} / 2-846 a_{3} c_{2}^{3}+963 c_{2}^{4} c_{3}^{2} / 4 \\
& -153 a_{3}^{2} c_{1} c_{3}^{3} / 2+9 c_{0} c_{1} c_{3}^{3} / 2+228 a_{3} c_{1} c_{2} c_{3}^{3}-333 c_{1} c_{2}^{2} c_{3}^{3} / 2 \\
& -c_{3}^{4}+357 a_{3}^{3} c_{3}^{4} / 2+12 a_{3} c_{0} c_{3}^{4}-9 c_{1}^{2} c_{3}^{4}-720 a_{3}^{2} c_{2} c_{3}^{4} \\
& -27 c_{0} c_{2} c_{3}^{4}+951 a_{3} c_{2}^{2} c_{3}^{4}-1647 c_{2}^{3} c_{3}^{4} / 4+165 a_{3} c_{1} c_{3}^{5} / 2 \\
& -108 c_{1} c_{2} c_{3}^{5}-519 a_{3}^{2} c_{3}^{6} / 4-27 c_{0} c_{3}^{6} / 2+294 a_{3} c_{2} c_{3}^{6}-639 c_{2}^{2} c_{3}^{6} / 4 \\
& -27 c_{1} c_{3}^{7} / 2+21 a_{3} c_{3}^{8} / 2-9 c_{2} c_{3}^{8} / 4+9 c_{3}^{10} / 4=0 .
\end{aligned}
$$

Further exclusion of variables required too much computer memory. So a complete search was used instead for $F=\mathbb{Z} / 7 \mathbb{Z}$ and $F=\mathbb{Z} / 13 \mathbb{Z}$. The author thanks A. Ocneanu for help with programming. The computer search showed that there was no solution for this system of five polynomial equations in five variables in the case $F=\mathbb{Z} / 7 \mathbb{Z}$. Thus, the equation $d=a^{3}+b^{3}+c^{3}$ has no solutions in $(\mathbb{Z} / 7 \mathbb{Z})[x]$ such that $5 \geq \operatorname{deg}(a) \geq \operatorname{deg}(b) \geq \operatorname{deg}(c), \operatorname{deg}(a) \neq 0$, and $\operatorname{deg}(d)=0$ or 1 . In the case $F=\mathbb{Z} / 13 \mathbb{Z}$, the computer took 57128 seconds to try all $13^{5}$ possible solutions and found all 12 solutions. The first of them is $\left(1+3 x+10 x^{2}+x^{5}\right)^{3}-\left(12+12 x+6 x^{2}+x^{5}\right)^{3}+\left(2+4 x+8 x^{2}+9 x^{3}+x^{4}\right)^{3}=10+8 x$. 
It was written above in the proof of Theorem 4 after a linear change of variable. Here are five more solutions $(\bmod 13)$ :

$$
\begin{aligned}
& \left(3+x+10 x^{2}+x^{5}\right)^{3}-\left(10+4 x+6 x^{2}+x^{5}\right)^{3} \\
& \quad+\left(5+4 x+11 x^{2}+3 x^{3}+x^{4}\right)^{3}=10+11 x \\
& \left(9+9 x+10 x^{2}+x^{5}\right)^{3}-\left(4+10 x+6 x^{2}+x^{5}\right)^{3} \\
& \quad+\left(6+4 x+7 x^{2}+x^{3}+x^{4}\right)^{3}=10+7 x ; \\
& \left(5+7 x+7 x^{2}+7 x^{3}+x^{5}\right)^{3}+\left(7+3 x+7 x^{2}+3 x^{3}+x^{4}\right)^{3} \\
& \quad-\left(12+10 x+3 x^{2}+7 x^{3}+x^{5}\right)^{3}=1 ; \\
& \left(2+11 x+7 x^{2}+8 x^{3}+x^{5}\right)^{3}-\left(10+12 x+3 x^{2}+8 x^{3}+x^{5}\right)^{3} \\
& \quad+\left(11+3 x+8 x^{2}+x^{3}+x^{4}\right)^{3}=1 ; \\
& \left(6+8 x+7 x^{2}+11 x^{3}+x^{5}\right)^{3}-\left(4+4 x+3 x^{2}+11 x^{3}+x^{5}\right)^{3} \\
& \quad+\left(8+3 x+11 x^{2}+9 x^{3}+x^{4}\right)^{3}=1 .
\end{aligned}
$$

The other six equalities can be obtained from the above six solutions by switching $a$ and $b$ and replacing $x$ by $-x$.

The degree- 6 case was treated similarly. The following lemma was used to restrict possible values for the coefficients of $a, b, c$.

Lemma 5. Let $F$ be a field such that there is no $\alpha, \beta, \gamma$ in $F$ with $\alpha^{3}+\beta^{3}+\gamma^{3}=$ $0 \neq \alpha \beta \gamma$. Let $x=a^{3}+b^{3}+c^{3}$ with $a, b, c \in F[x], N=\operatorname{deg}(a) \geq \operatorname{deg}(b) \geq$ $\operatorname{deg}(c) \geq 1, a=\sum a_{i} x^{i}, b=\sum b_{i} x^{i}, c=\sum c_{i} x^{i}$, and $a_{i}, b_{i}, c_{i} \in F$. Then $c_{0}=0$ and $a_{0} / a_{N}$ is a cube in $F$.

Proof. By the condition, $c_{N}=0$, hence $\left(-b_{N} / a_{N}\right)^{3}=1$. Replacing $b$ by $b b_{N} / a_{N}$, we can assume that $b_{N}=-a_{N} \neq 0$.

Let us show that the assumption that $c_{0} \neq 0$ leads to a contradiction. Indeed, in this case $a_{0} b_{0}=0$. Say, $b_{0}=0$. Replacing $c$ by $c a_{0} / c_{0}$ we can assume that $c_{0}=a_{0}$.

Let $\alpha_{i}$ be the zeros of $b+c$ in a field extension of $F$. Then $a\left(\alpha_{i}\right)^{3}=\alpha_{i}$ for all $i$, hence $a_{0} / a_{N}=(-1)^{N} \prod \alpha_{i}=\left((-1)^{N} \prod a\left(\alpha_{i}\right)\right)^{3}$ is a cube in $F$. Applying this argument to the zeros $\beta_{j}$ of $a^{2}-a b+b^{2}$, we obtain that $a_{0}^{2} /\left(3 a_{N}^{2}\right)=$ $\left(\prod c\left(\beta_{j}\right)\right)^{3}$ is also a cube in $F$. So 9 is a cube (in fact, a 12th power) in $F$. In the case $2 F=F$, this leads to a contradiction, because $9=8+1$ is also the sum of two cubes.

In the case $2 F=0$, assume first that $F$ contains an element $\varepsilon$ such that $\varepsilon^{2}=\varepsilon+1$. Using the zeros of $a+\varepsilon b$, we conclude that $a_{0} /\left(a_{N}(1+\varepsilon)\right)$ is a cube in $F$. Using the zeros of $a+\varepsilon c$, we conclude that $a_{0}(1+\varepsilon) / a_{N}$ is a cube in $F$. So $(1+\varepsilon)^{2}=\varepsilon$ is a cube in $F$. This leads to a contradiction, because $\varepsilon=1+1 / \varepsilon$ is also the sum of two cubes.

Assume now that $2 F=0$ and $F$ does not contain any $\varepsilon$ as above. Then we consider a field extension $F[\varepsilon]$ with $\varepsilon$ as above. Using the zeros of $a+\varepsilon b$, 
we conclude that $a_{0} /\left(a_{N}(1+\varepsilon)\right)$ is a cube in $F[\varepsilon]$. Using the zeros of $a+\varepsilon c$, we conclude that $a_{0}(1+\varepsilon) / a_{N}$ is a cube in $F[\varepsilon]$. So $(1+\varepsilon)^{2}=\varepsilon$ is a cube in $F[\varepsilon]$. We write $\varepsilon=(u+v \varepsilon)^{3}$ with $u, v \in F$. Then

$$
0=u^{3}+u v^{2}+v^{3}=(u+v)^{3}-u^{2} v \text { and } \quad 1=u^{2} v+u v^{2}=u v(u+v),
$$

hence $u^{5} v^{4}=1$. Combining this with

$$
0=\left(1+u^{2} v+u v^{2}\right)^{4}=1+u^{8} v^{4}+u^{4} v^{8}
$$

we obtain that $1+u^{3}+u^{-6}=0$, which contradicts the condition of the lemma.

Thus, $c_{0}=0$ in all cases. Now we use the zeros $\gamma_{k}$ of $a+c$ to conclude that $a_{0} / a_{N}=\left((-1)^{N} \prod b\left(\gamma_{k}\right)\right)^{3}$ is a cube in $F$.

Proof of Proposition 3. It was shown above that the condition of Lemma 1 implies that $x$ is the sum of cubes of three polynomials of degree 1 .

Assume now that there is no $\alpha, \beta, \gamma$ in $F=6 F$ such that $\alpha^{3}+\beta^{3}+\gamma^{3}=$ $0 \neq \alpha \beta \gamma$. We want to prove that $\operatorname{deg}(d) \neq 1$ for any

$$
d=a^{3}+b^{3}+c^{3},
$$

where $a, b, c \in F[x]$, provided that $a, b, c$ are all of degree $\leq 4$.

Let $\operatorname{deg}(a) \geq \operatorname{deg}(b) \geq \operatorname{deg}(c)$. Assume that $\operatorname{deg}(d)=1$. Then it is clear that all three polynomials $a, b, c$ cannot have the same degree. So $\operatorname{deg}(a)=$ $\operatorname{deg}(b)>\operatorname{deg}(c)$. Dividing both sides of (6) by the cube of the leading coefficient of $a$, we can assume that $a$ and $-b$ are monic, i.e., their leading coefficients are 1 . Then the next two coefficients must also be equal. This leads immediately to a contradiction when the degree of $a$ is $\leq 2$.

Consider now the case when $\operatorname{deg}(a)=3$ or 4 . If $2 F=F$, we set $u=a+b$ and $v=a-b$. The equation takes the form

$$
u\left(u^{2}+3 v^{2}\right) / 4+c^{3}=d .
$$

When $\operatorname{deg}(a)=3$, we see that $\operatorname{deg}(c)=2, \operatorname{deg}(u)=0$, and $\operatorname{deg}(v)=3$. We rewrite the equation as $v^{2} / 4+c^{3} / 3 u=d / 3 u-u^{2} / 3=d^{\prime}$ with $\operatorname{deg}\left(d^{\prime}\right)=1$. We write $v / 2=x^{3}+v_{2} x^{2}+v_{1} x+v_{0}$ and $c^{3} / 3 u=-\left(x^{2}+c_{1} x+c_{0}\right)^{3}$. Our equation takes the form

$$
\left(x^{3}+v_{2} x^{2}+v_{1} x+v_{0}\right)^{2}-\left(x^{2}+c_{1} x+c_{0}\right)^{3}=d^{\prime} .
$$

Then we replace $x$ by $x-v_{2} / 3$ to make $v_{2}=0$. Looking at terms of degree 5 , we conclude that $c_{1}=0$. Looking at terms of degree 3 , we conclude that $v_{0}=0$. Looking at terms of degree 1 , we obtain a contradiction. (The " $a b c$ theorem" yields that $V^{2}-C^{3}$ cannot be a nonzero constant for nonconstant polynomials $V$ and $C$, if $\operatorname{char}(F)=0$. It is not difficult to show that the necessary and sufficient condition on a field $F$ for this conclusion to be true is that $6 F=F$.) 
Let now $\operatorname{deg}(a)=4$ (and still $2 F=F$ ). Since $\operatorname{deg}(v)=4$, it is clear that $\operatorname{deg}(u)=1$ and $\operatorname{deg}(c)=3$. Now we set $y=1 / u$. After we divide both sides of (6) by $u^{9}$, it takes the form

$$
3 / 4\left(v / u^{4}\right)^{2}+\left(c / u^{3}\right)^{3}=-y^{6} / 4+x / u^{9} .
$$

Note that $c / u^{3}$ is a polynomial of degree 3 in $y$ with a constant term $e^{\prime} \neq 0$. After dividing the equation by $e^{\prime 3}$, it takes the form

$$
\begin{aligned}
& \left(1+v_{1} y+v_{2} y^{2}+v_{3} y^{3}+v_{4} y^{4}\right)^{2}-\left(1+c_{1} y+c_{2} y^{2}+c+3 y^{3}\right)^{3} \\
& \quad=-y^{6} / 4 e^{\prime 3}+e^{\prime \prime} y^{8}+e^{\prime \prime \prime} y^{9} \quad \text { with } e^{\prime}, e^{\prime \prime}, e^{\prime \prime \prime} \text { in } F \text { and } e^{\prime} e^{\prime \prime} \neq 0 .
\end{aligned}
$$

Now we see that, modulo $y^{6} F[y]$,

$$
\left(1+v_{1} y+v_{2} y^{2}+v_{3} y^{3}+v_{4} y^{4}\right)^{2} \equiv\left(1+c_{1} y+c_{2} y^{2}+c_{3} y^{3}\right)^{3},
$$

hence

$$
1+v_{1} y+v_{2} y^{2}+v_{3} y^{3}+v_{4} y^{4} \equiv\left(1+f_{1} y+f_{2} y^{2}+f_{3} y^{3}+f_{4} y^{4}+f_{5} y^{5}\right)^{3}
$$

and

$$
1+c_{1} y+c_{2} y^{2}+c_{3} y^{3} \equiv\left(1+f_{1} y+f_{2} y^{2}+f_{3} y^{3}+f_{4} y^{4}+f_{5} y^{5}\right)^{2}
$$

for some $f_{i}$ in $F$. Set $f=1+f_{1} y+f_{2} y^{2}+f_{3} y^{3}+f_{4} y^{4}+f_{5} y^{5}$.

Then, modulo $y^{6} F[y]$,

$$
\begin{aligned}
f^{2} \equiv & 1+2 f_{1} y+\left(2 f_{2}+f_{1}^{2}\right) y^{2}+\left(2 f_{3}+2 f_{1} f_{2}\right) y^{3} \\
& +\left(2 f_{4}+2 f_{1} f_{3}+f_{2}^{2}\right) y^{4}+\left(2 f_{5}+2 f_{1} f_{4}+2 f_{2} f_{3}\right) y^{5}
\end{aligned}
$$

and

$$
\begin{aligned}
f^{3} \equiv & 1+3 f_{1} y+\left(3 f_{2}+3 f_{1}^{2}\right) y^{2}+\left(3 f_{3}+6 f_{1} f_{2}+f_{1}^{3}\right) y^{3} \\
& +\left(3 f_{4}+3 f_{1}^{2} f_{2}+6 f_{1} f_{3}+3 f_{2}^{2}\right) y^{4} \\
& +\left(3 f_{5}+6 f_{1} f_{4}+6 f_{2} f_{3}+3 f_{1}^{2} f_{3}+3 f_{1} f_{2}^{2}\right) y^{5} .
\end{aligned}
$$

So

$$
\begin{aligned}
& 0=c_{4}=2 f_{4}+2 f_{1} f_{3}+f_{2}^{2}, \\
& 0=c_{5}=2 f_{5}+2 f_{1} f_{4}+2 f_{2} f_{3}, \\
& 0=v_{5}=3 f_{5}+3 f_{1} f_{2}^{2}+6 f_{1} f_{4}+6 f_{2} f_{3}+3 f_{1}^{2} f_{3},
\end{aligned}
$$

hence

$$
f_{4}+f_{2}^{2} / 2+f_{3} f_{1}=0, \quad f_{5}-f_{2}^{2} f_{1}-f_{3} f_{1}^{2}=0, \quad f_{3} f_{2}+f_{2}^{2} f_{1} / 2=0 .
$$

We set $C=1+2 f_{1} y+\left(2 f_{2}+f_{1}^{2}\right) y^{2}+\left(2 f_{3}+2 f_{1} f_{2}\right) y^{3}$,

$$
\begin{aligned}
V=1 & +3 f_{1} y+\left(3 f_{2}+3 f_{1}^{2}\right) y^{2}+\left(3 f_{3}+6 f_{1} f_{2}+f_{1}^{3}\right) y^{3} \\
& +\left(3 f_{4}+3 f_{1}^{2} f_{2}+6 f_{1} f_{3}+3 f_{2}^{2}\right) y^{4},
\end{aligned}
$$


and $S=V^{2}-C^{3}$. Then (7) takes the form

$$
\begin{aligned}
S & =V^{2}-C^{3}=S_{5} y^{5}+S_{6} y^{6}+S_{7} y^{7}+S_{8} y^{8}+S_{9} y^{9} \\
& =-y^{6} / 4 e^{\prime 3}+e^{\prime \prime} y^{8}+e^{\prime \prime \prime} y^{9}
\end{aligned}
$$

where the coefficients $S_{i}$ of $S$ are polynomials in $f_{j}$. Substituting $f_{4}=$ $-f_{2}^{2} / 2-f_{33} f_{1}$ and $f_{5}=f_{2}^{2} f_{1}+f_{3}^{3} f_{1}^{2}$ into $S_{i}$, we obtain:

$$
\begin{aligned}
S_{9}= & -8 f_{3}^{3}-24 f_{3}^{2} f_{2} f_{1}-24 f_{3} f_{2}^{2} f_{1}^{2}-8 f_{2}^{3} f_{1}^{3}=e^{\prime \prime \prime}, \\
S_{8}= & -24 f_{3}^{2} f_{2}+9 f_{2}^{4} / 4-39 f_{3} f_{2}^{2} f_{1}-3 f_{3}^{2} f_{1}^{2} \\
& -15 f_{2}^{3} f_{1}^{2}-6 f_{3} f_{2} f_{1}^{3}-3 f_{2}^{2} f_{1}^{4}=e^{\prime \prime} \neq 0, \\
S_{7}= & -15 f_{3} f_{2}^{2}-6 f_{3}^{2} f_{1}-6 f_{2}^{3} f_{1}-18 f_{3} f_{2} f_{1}^{2}-9 f_{2}^{2} f_{1}^{3}=0, \\
S_{6}= & -3 f_{3}^{2}+f_{2}^{3}-18 f_{3} f_{2} f_{1}-9 f_{2}^{2} f_{1}^{2}=-1 / 4 e^{\prime 3} \neq 0, \\
S_{5}= & -6 f_{3} f_{2}-3 f_{2}^{2} f_{1}=0 .
\end{aligned}
$$

Since $S_{5}=0$, we see that either $f_{2}=0$, or $2 f_{3}+f_{1} f_{2}=0$.

In the first case,

$$
\left(S_{9}, S_{8}, S_{7}, S_{6}\right)=\left(-8 f_{3}^{3},-3 f_{3}^{2} f_{1}^{2},-6 f_{3}^{2} f_{1},-3 f_{3}^{2}\right),
$$

and $S_{7}=-6 f_{3}^{2} f_{1}=0$ implies that $S_{8}=-3 f_{3}^{2} f_{1}^{2}=0$, so this case is impossible.

Assume now that $f_{2} \neq 0=2 f_{3}+f_{1} f_{2}$. Then

$$
\begin{gathered}
S_{9}=-f_{2}^{3} f_{1}^{3}, \quad S_{8}=9 f_{2}^{4} / 4-3 f_{2}^{3} f_{1}^{2} / 2-3 f_{2}^{2} f_{1}^{4} / 4, \\
S_{7}=3 f_{2}^{3} f_{1} / 2-3 f_{2}^{2} f_{1}^{3} / 2, \quad S_{6}=f_{2}^{3}-3 f_{2}^{2} f_{1}^{2} / 4 .
\end{gathered}
$$

From $S_{7}=3 f_{2}^{3} f_{1} / 2-3 f_{2}^{2} f_{1}^{3} / 2=0$, we obtain that either $f_{1}=0$, or $f_{2}=f_{1}^{2}$. The latter case is impossible because then the coefficient $S_{7}$ vanishes. So let $f_{1}=0$.

Then the coefficients $\left(S_{9}, S_{8}, S_{7}, S_{6}\right)$ become $\left(0,9 f_{2}^{4} / 4,0, f_{2}^{3}\right)$. So $-e^{\prime 3} / 4$ $=f_{2}^{3}$, hence 2 is a cube in $F$. Since 2 is also the sum of two cubes, we are done.

The case $2 F=0, \operatorname{deg}(a)=3$ or 4 was done using the computer. When $\operatorname{deg}(a)=3$, we consider (6) with $a=x^{3}+a_{2} x^{2}+a_{1} x+a_{0}, b=a+f$, $c=c_{2} x^{2}+c_{1} x+c_{0}, d=\sum d_{i} x^{i} \neq 0, d_{i}=0$ for $i \geq 2$.

It is clear that $\operatorname{deg}(f)=0$. Replacing $x$ by $x+c_{2}$, we make $c_{2}=0$. From $d_{6}=f+c_{2}^{3}=0$, we get $f=c_{2}^{3}$. Substituting this into $d$, we get that $d_{5}=c_{1} c_{2}^{2}=0$. If $c_{1}=0$, then $d_{3}=c_{2}^{6}=0$. Thus, $c_{2}=0$, hence $d_{2}=c_{0} c_{1}^{2}$ and $d_{1}=c_{0}^{2} c_{1}$. So $d_{2}=0$ implies that $d_{1}=0$.

The reader is spared from longer computations which were done in the case when $2 F=0$ and $\operatorname{deg}(a)=4$.

Remark. The identity

$$
x=\left(\left(x^{3}-1 / 27\right)^{3}+\left(x^{2}+x / 3\right)^{3}+\left(-x^{3}+x / 3+1 / 27\right)^{3}\right) /\left(x^{2}+x / 3+1 / 9\right)^{3}
$$


shows that $w_{3}(F(x)) \leq 3$ and $w_{3}(F) \leq 3$ for any field $F$ with $\operatorname{char}(F) \neq 3$; if $\operatorname{char}(F)=3$, then $w_{3}(F(x))=w_{3}(F)=1$.

Remarks. All the solutions of $(6)$ with $\operatorname{deg}(a)=\operatorname{deg}(b)=3, \operatorname{deg}(c)=2, \operatorname{deg}(d)$ $=0$ can be obtained from

$$
\left(1+6 x^{3}\right)^{3}+\left(1-6 x^{3}\right)^{3}+\left(-6 x^{2}\right)^{3}=2
$$

by a linear change of variable and scaling. There are no such solutions when $2 F=0$.

All the solutions of (6) with $\operatorname{deg}(a)=\operatorname{deg}(b)=4, \operatorname{deg}(c)=3, \operatorname{deg}(d)=0$ can be obtained from

$$
\left(9 x^{4}\right)^{3}+\left(3 x-9 x^{4}\right)^{3}+\left(1-9 x^{3}\right)^{3}=1
$$

by a linear change of variable, switching $a$ and $b$, and scaling. P. Erdös pointed out to the author that the last two formulas can be found in [1].

All solutions of (6) with $\operatorname{deg}(a)=\operatorname{deg}(b)=5, \operatorname{deg}(c)=4, \operatorname{deg}(d)=0$ or 1 were described above when $\operatorname{char}(F)=13$. They do not exist when $\operatorname{card}(F)=5,7$. When $\operatorname{char}(F)=11$, all such solutions can be obtained from the equalities

$$
\begin{gathered}
\left(2+2 x^{2}+3 x^{3}+x^{5}\right)^{3}-\left(2+5 x+6 x^{2}+3 x^{3}+x^{5}\right)^{3} \\
+\left(x+10 x^{2}+5 x^{3}+x^{4}\right)^{3}=6 x \\
\left(1+4 x+x^{2}+5 x^{3}+x^{5}\right)^{3}-\left(9+6 x+5 x^{2}+5 x^{3}+x^{5}\right)^{3} \\
+\left(8+x+2 x^{3}+x^{4}\right)^{3}=4 \\
\left(4+4 x+3 x^{2}+8 x^{3}+x^{5}\right)^{3}-\left(8+6 x+7 x^{2}+8 x^{3}+x^{5}\right)^{3} \\
+\left(5+4 x+9 x^{2}+2 x^{3}+x^{4}\right)^{3}=7
\end{gathered}
$$

by a linear change of variable, switching $a$ and $b$, and scaling.

\section{BIBLIOGRAPHY}

1. K. Mahler, Note on hypothesis K of Hardy and Littlewood, J. London Math. Soc. 11 (1936), 136-138.

2. L. J. Mordell, On representation of an integer as sum of four integral cubes, Computers in Number Theory, Academic Press, 1971.

3. R. E. A. C. Paley, Theorems on polynomials in a Galois field, Quart. J. Math. 4 (1933), 52-63.

4. L. N. Vaserstein, Waring's problem for algebras over fields, J. Number Theory 26 (1987), 286-298.

Department of Mathematics, The Pennsylvania State University, University Park, PenNSYlVANIA 16802

E-mail address: vstein(a)math.psu.edu 